\title{
Comparative study of electronic and magnetic properties of iron and cobalt phthalocyanine molecules physisorbed on two-dimensional $\mathrm{MoS}_{2}$ and graphene
}

\author{
Soumyajyoti Haldar, ${ }^{1, *}$ Sumanta Bhandary, ${ }^{2}$ Hakkim Vovusha,,${ }^{1, \dagger}$ and Biplab Sanyal ${ }^{1}$ \\ ${ }^{1}$ Division of Materials Theory, Department of Physics and Astronomy, Uppsala University, Box-516, SE 75120, Sweden \\ ${ }^{2}$ CPHT, Ecole Polytechnique, CNRS, Université Paris-Saclay, Route de Saclay, 91128 Palaiseau, France
}

(Received 21 March 2018; revised manuscript received 6 July 2018; published 31 August 2018)

\begin{abstract}
In this paper, we have done a comparative theoretical study of electronic and magnetic properties of iron phthalocyanine $(\mathrm{FePc})$ and cobalt phthalocyanine $(\mathrm{CoPc})$ molecules physisorbed on a monolayer of $\mathrm{MoS}_{2}$ and graphene by density functional theory. Various types of physisorption sites have been considered for both surfaces. The lowest energy structure for both metal phthalocyanine (MPc) molecules physisorbed on $\mathrm{MoS}_{2}$ is a sulfur-top position, i.e., when the metal center of the molecule is on top of a sulfur atom. However, on graphene, the lowest energy structure for the FePc molecule is when a metal atom is on top of a bridge position. In contrast to this, the CoPc molecule prefers a carbon-top position. The adsorption of $M \mathrm{Pc}$ molecules is stronger on the $\mathrm{MoS}_{2}$ surface than on graphene ( $\sim 2.5 \mathrm{eV}$ higher physisorption energy). In these systems, spin dipole moments of the metal centers are antiparallel to the spin moments and hence a huge reduction of effective spin moment can be seen. The calculations of magnetic anisotropy energies using both variational and second-order perturbation approaches indicate no significant changes after physisorption. In case of the FePc and CoPc physisorption, respectively, an out-of-plane and an in-plane easy axis of magnetization can be observed. Our calculations indicate a reduction of $\mathrm{MoS}_{2}$ work function $\sim 1 \mathrm{eV}$ due to physisorption of $M \mathrm{Pc}$ molecules while it does not change significantly in the case of graphene.
\end{abstract}

DOI: 10.1103/PhysRevB.98.085440

\section{INTRODUCTION}

Over the past two decades, the research fields of molecular electronics and spintronics have achieved a significant advancement with a remarkable progress in nanoscience, particularly the development of experimental techniques such as scanning tunneling microscopy, mechanically controlled break junctions, amd electromigration break junctions, which has made it possible to detect, manipulate, and utilize intrinsic single molecular properties for electronic and spintronic device fabrication. Integrability of a molecule in a device without compromising its intrinsic properties is a key challenge on this front and sets the current trend of research in molecular electronics and spintronics. Metal-organic complexes are particularly potent from these aspects, as extended organic complex plays a major role in device integrability while the central ionic complex carries out the key molecular features. However, to achieve the functionalities of electronic (spintronic) device, molecules are required to express multiple conformations, be it structural or electronic, that can be accessed externally. Metal-porphyrin $(M \mathrm{P})$ and -phthalocyanine $(M \mathrm{Pc})$ molecules hold an immense potential

\footnotetext{
*Present address: Institute of Theoretical Physics and Astrophysics, University of Kiel, Leibnizstrasse 15, 24098 Kiel, Germany; Corresponding author: haldar@physik.uni-kiel.de, haldar.physics@gmail.com

${ }^{\dagger}$ Present address: King Abdullah University of Science and Technology (KAUST), Physical Science and Engineering Division (PSE), Thuwal 23955-6900, Saudi Arabia.
}

in this regard, as they offer conformation switching based on structural [1], charge [2], and spin degrees [3] of freedom. MP and $M \mathrm{Pc}$ molecules containing magnetic core are of particular interest for spin-based molecular devices. The molecules often respond to the external manipulations, such as temperature, light, or mechanical strain, and exhibit spin-crossover or change in magnetic anisotropy [4]. Both these properties are extremely crucial for molecule-based spin valve or magnetic storage devices. In addition, these molecules posses planar structures, which improve significantly the feasibility of adsorption on different surfaces and are found to have longrange structural ordering [5]. These features pave the way to exploit the interface properties of molecules and substrates to control the molecular properties for desired applications. Several fundamental approaches based on magnetic properties of $M \mathrm{P}$ and $M \mathrm{Pc}$ have been explored both experimentally and theoretically. The manipulation of the spin state with surface-controlled ligand field [6,7], molecule-surface magnetic exchange coupling with surface modification $[8,9]$ and additional ligation of functional groups [10], or emergence of Kondo resonance [11] have been reported, emphasizing the accessibility of the magnetic states in these molecules.

However, the choice of adsorbent surface is equally crucial for controlling the molecular properties as it dictates the interface properties. Atomically thin layers are particularly appealing from this aspect with their exotic surface properties and also from the point of view in finding applications in nanoscale devices. The absorption properties of ultrathin graphene, graphene-BN layers, have been explored extensively [12-14], which combined with intrinsic molecular properties [6] raise the potential for the designing of devices. 
Two-dimensional (2D) $\mathrm{MoS}_{2}$ is an ultrathin semiconductor with unique electronic and optical properties, that has potential applications in optoelectronics, fluorescence imaging, photo catalysis, solar cells, valley electronics or as photodynamic, photo thermal materials and even in biomedicine as antibacterial agents [15-21]. Monolayer $\mathrm{MoS}_{2}$ has a direct band gap of $1.9 \mathrm{eV}$, which is suitable to absorb visible light [22,23]. The multifunctionalities of $\mathrm{MoS}_{2}$ have opened up many new routes to encompass the interface manipulations. Tuning of electronic and optical properties of $\mathrm{MoS}_{2}$ by means of molecular charge transfer using organic molecules adsorption has also been reported in recent theoretical studies [24]. Effects of $\mathrm{CuPc}$ and TiOPc physisorption on the optical properties of 2D $\mathrm{MoS}_{2}$ have also been investigated recently [25].

In this paper, we have explored extensively the physisorption properties of $M \mathrm{Pc}$ molecules on single free-standing layers of $\mathrm{MoS}_{2}$ and graphene and their impact on the electronic and specifically the magnetic properties of the molecule, aiming at their applications in spintronic devices. FePc and $\mathrm{CoPc}$ molecules were chosen as the representatives of metal phthalocyanine molecules, owing to the subtle balance between spin-pairing energy and ligand field that gives rise to exotic electronic and magnetic properties in the molecules. Our calculations indicate a stronger adsorption of $M \mathrm{Pc}$ on $\mathrm{MoS}_{2}$ surface compared to that on a graphene layer, along with different favored adsorption sites. With the aid of density functional theory, incorporating static electron correlation $(\mathrm{DFT}+\mathrm{U})$, we've performed a detailed analysis of molecular adsorption scenario, surface work functions, and the impact of the interface properties on the electronic and magnetic structures of the molecules. The plan of the paper is as follows. In the next section, we will present the computational details followed by Results and Discussions in Sec. III. In the Results and Discussions section, we will first discuss the structural properties (Sec. III A), and then it will be followed by the discussion of electronic structure (Sec. IIIB), spin dipole contributions (Sec. III B 2), magnetic anisotropy (Sec. III B 3), and work function (Sec. III B 4). The conclusions are presented in Sec. IV.

\section{COMPUTATIONAL DETAILS}

All the calculations have been performed with supercells of monolayer $\mathrm{MoS}_{2}$ and graphene. The supercells of $\mathrm{MoS}_{2}$ and graphene are generated by repeating the primitive cells by 9 and 12 times, respectively, in both $a$ and $b$ directions. The $M \mathrm{Pc}(M=\mathrm{Fe}, \mathrm{Co})$ molecules are placed on top of these surfaces. A vacuum of $20 \AA$ is included to avoid the interaction with periodic images. All the calculations have been performed using plane-wave-based density functional code VASP [26] within the projector augmented wave method (PAW) for describing electron-ion interactions. The generalized gradient approximation (GGA) of Perdew, Burke, and Ernzerhof (PBE) has been used for the exchange-correlation potential $[27,28]$. All the structures have been fully relaxed using the conjugate gradient method with the forces calculated using the Hellman-Feynman theorem. We have used the energy and the Hellman-Feynman force thresholds of $10^{-4}$ $\mathrm{eV}$ and $10^{-2} \mathrm{eV} / \AA$, respectively. We have used $500 \mathrm{eV}$ cutoff energy to truncate the plane waves. A $3 \times 3 \times 1$ Monkhorst-
Pack $k$-grid was used for all our calculations. To address the problem of electron correlation effect in the narrow $d$ states of metal atoms $(\mathrm{Fe}, \mathrm{Co})$, we have used $\mathrm{GGA}+\mathrm{U}$ approach following the formalism proposed by Dudarev et al. [29] A Coulomb interaction term is added according to the mean field Hubbard U formalism [29-31]. For the exchange parameter, J, we have used a value of $1.0 \mathrm{eV}$. We have used $4.0 \mathrm{eV}$ and 6.0 $\mathrm{eV}$ as the Coulomb parameter, $\mathrm{U}$, values for $\mathrm{FePc}$ and $\mathrm{CoPc}$, respectively $[32,33]$. These values are taken as they correctly reproduce the electronic structures and magnetic properties of $\mathrm{FePc}$ and $\mathrm{CoPc}$ in gas phase.

The van der Waals (vdW) interaction between the MPc molecules and the $\mathrm{MoS}_{2}$ or graphene layer originates due to the fluctuating charge distribution and hence cannot be described by PBE functionals alone. Hence for all the calculations, we have included the vdW effect by adding a correction to the conventional Kohn-Sham DFT energy through a pair wise force field following the method proposed by Tkatchenko-Scheffler [34].

The physisorption energy, $E_{a}$, for metal phthalocyanine molecule physisorbed on $\mathrm{MoS}_{2}$ or graphene is defined as follows:

$$
E_{a}=\left[E_{\text {layer }}+E_{M \mathrm{Pc}}\right]-E_{\text {layer }+M \mathrm{Pc}},
$$

where

(i) $E_{\text {layer }}$ is the total energy of either $\mathrm{MoS}_{2}$ or graphene supercell.

(ii) $E_{M \mathrm{Pc}}$ is the total energy of the $M \mathrm{Pc}$ molecules in the gas phase.

(iii) $E_{\text {layer }+M \mathrm{Pc}}$ is the total energy of $M \mathrm{Pc}$ physisorbed on $\mathrm{MoS}_{2}$ or graphene supercell system.

These total energies are taken after the structures are fully relaxed, according to the force convergence criterion. Hence, they include the energy contributions due to any kind of structural deformations.

\section{RESULTS AND DISCUSSIONS}

\section{A. Structural properties}

We will begin our discussion of results from the analyses of structural properties and the energetics of the systems studied. We will first discuss the results of $M \mathrm{Pc}$ physisorption on $\mathrm{MoS}_{2}$ followed by the results of physisorption on graphene.

To find out the most energetically favorable physisorbed position, we have considered different possible positions of the MPc molecules on the 2D surfaces. These different positions of the molecules can be distinguished by the position of the metal atom center with respect to the underlying surface layer of $\mathrm{MoS}_{2}$ or graphene. For $\mathrm{MoS}_{2}$, there are four possible positions, which are (i) S-Top-when the metal atom center is at the top of an S atom, (ii) Mo-Top-when the metal atom center is at the top of a Mo atom of the second atomic layer, (iii) Bridge - when the metal atom center is at the bridge position of two $\mathrm{S}$ atoms belonging to the top layer of $\mathrm{MoS}_{2}$, and (iv) Hex - when the metal atom center is on top of the center of a hexagon, created by Mo and S. In Fig. 1, we have shown the representations of these four positions for $\mathrm{FePc}$ molecule on $\mathrm{MoS}_{2}$ only. However, these different geometry positions are also applicable for the CoPc molecule. In the case of graphene, however, only three positions are 

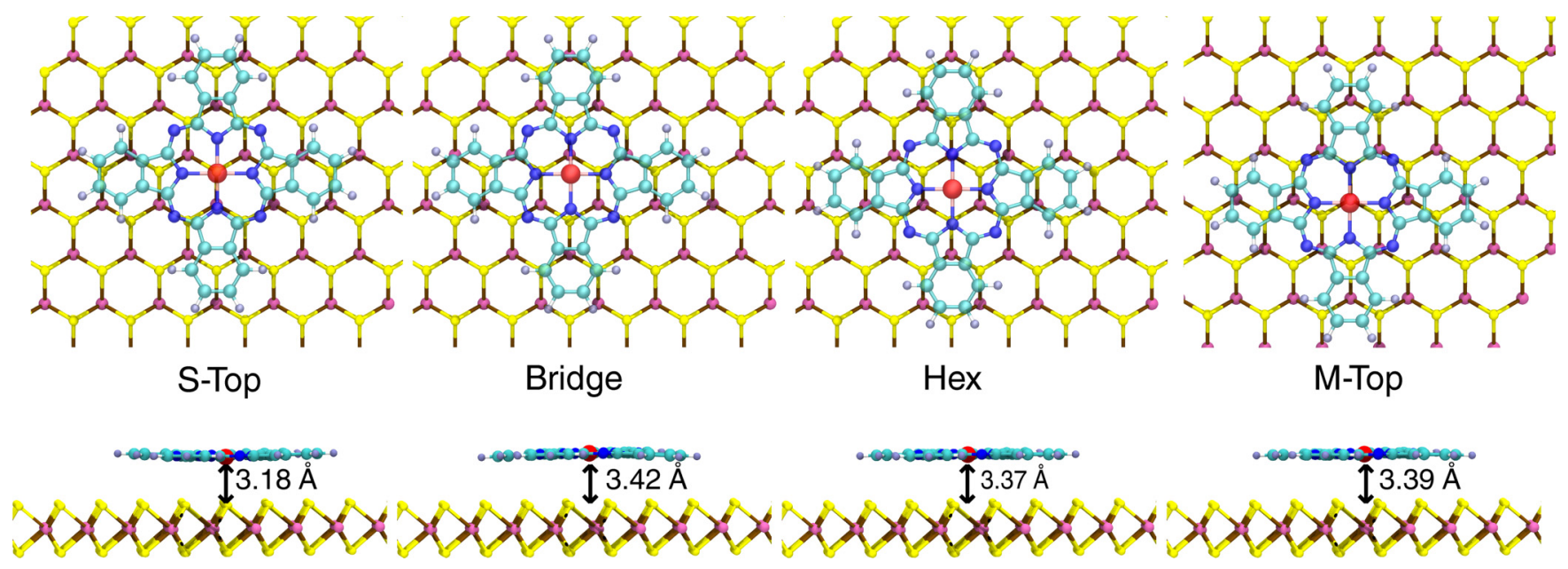

FIG. 1. Representative figure of various optimized geometries considered for $M P c$ molecules on $\mathrm{MoS}_{2}$ in our calculations to obtain energetically most favorable positions for physisorption. These geometry positions are S-Top, Bridge, Hexagonal, and M-Top (from left to right, respectively). We have taken FePc molecule on $\mathrm{MoS}_{2}$ as the representative to show these positions. The upper panel of the figure shows a closeup top view of these four structural positions. The lower panel shows the side view for the same along with the vertical distance from Fe atom to the top S layer. See Sec. III A for detailed discussions.

possible. Following the above terminology, these positions are (i) C-Top, (ii) Bridge, and (iii) Hex. In our calculations, $M P c$ molecules are placed on the surfaces with a particular $x y$ position and then we have relaxed the atomic positions completely without any constraint until the force convergence was achieved.

In Table I, we have shown the physisorption energies $\left(E_{a}\right)$ and the vertical distances $\left(D_{h}\right)$ between the metal center and the $\mathrm{MoS}_{2}$ layer for all of the four FePc and CoPc physisorbed structures as described before. The analyses of geometries reveal that for both molecules, all four bond-lengths between the metal and the nitrogen atoms are identical-1.95 $\AA$ for FePc and $1.94 \AA$ for CoPc, maintaining a $D_{4 h}$ symmetry of the molecule. For S-Top configuration, the vertical heights from the $\mathrm{MoS}_{2}$ layer are different for the two molecules. The vertical distances between the metal center of the molecules and the $\mathrm{MoS}_{2}$ layer are $3.18 \AA$ and 3.21 $\AA$ for FePc and CoPc, respectively. Interestingly, the vertical separations between the molecule and the $\mathrm{MoS}_{2}$ layer for the other geometry positions are almost identical. A close inspection reveals that the molecules are slightly concave upward and downward for S-Top and Bridge configurations, respectively. However,

TABLE I. Physisorption energies $\left(E_{a}\right)$ [see Eq. (1)] and vertical distances of $M$ atom $\left(\mathrm{D}_{h}\right)$ from top $\mathrm{S}$ layer of $\mathrm{MoS}_{2}$ for $M \mathrm{Pc}$ physisorbed on $\mathrm{MoS}_{2}$. The most stable structure with highest $E_{a}$ is written in bold. On $\mathrm{MoS}_{2}$, the vdW energy contributions for FePc and $\mathrm{CoPc}$ physisorptions are around three times larger than that on graphene.

\begin{tabular}{lccccc}
\hline \hline $\begin{array}{l}\text { FePc@ } \\
\mathrm{MoS}_{2}\end{array}$ & $\begin{array}{c}\mathrm{E}_{a} \\
(\mathrm{eV})\end{array}$ & $\begin{array}{c}\mathrm{D}_{h} \\
(\AA)\end{array}$ & $\begin{array}{c}\text { CoPc@ } \\
\mathrm{MoS}_{2}\end{array}$ & $\begin{array}{c}\mathrm{E}_{a} \\
(\mathrm{eV})\end{array}$ & $\begin{array}{c}\mathrm{D}_{h} \\
(\AA)\end{array}$ \\
\hline S-Top & $\mathbf{6 . 2 1}$ & $\mathbf{3 . 1 8}$ & S-Top & $\mathbf{6 . 1 8}$ & $\mathbf{3 . 2 1}$ \\
Bridge & 6.06 & 3.42 & Bridge & 6.05 & 3.42 \\
Hex & 6.03 & 3.37 & Hex & 6.01 & 3.38 \\
Mo-Top & 5.98 & 3.39 & Mo-Top & 5.96 & 3.40 \\
\hline \hline
\end{tabular}

the molecules remain flat for the other two configurations. The physisorption energies for S-Top geometry configurations for both FePc and $\mathrm{CoPc}$ are at least $\sim 0.14 \mathrm{eV}$ higher as compared to that of the other configurations (see Table I). The lowest physisorption energies can be observed for MoTop configurations. These are mainly because $\mathrm{S}$ atom is more electronegative as compared to that of Mo atom and interacts with the metal atom center of the MPc molecule more strongly. This fact is also evident from the concave and flat molecular structure of MPc molecules in S-Top and Mtop configurations, respectively. Hence, we can conclude that the S-Top configuration is energetically the most favorable physisorption structure for the MPc molecules physisorbed on $\mathrm{MoS}_{2}$.

We have also calculated the physisorption of the $M \mathrm{Pc}$ molecules on graphene to compare the same on $\mathrm{MoS}_{2}$. To find out the most energetically favorable physisorption sites, we have optimized three different geometry configurations of $M P c$ molecules physisorbed on graphene.

The calculated physisorption energies $\left(E_{a}\right)$ and vertical distances between the metal center and graphene layer $\left(D_{h}\right)$ are tabulated in Table II. The analyses of physisorption energies show that for graphene, the most favorable site for FePc molecule physisorption is when the Fe metal center resides at the bridge position (figure not shown). This is quite different

TABLE II. Physisorption energies $\left(E_{a}\right)$ [see Eq. (1)] and vertical distances of $M$ atom $\left(\mathrm{D}_{h}\right)$ from graphene layer for $M \mathrm{Pc}$ physisorbed on graphene. The most stable structure with highest $E_{a}$ is written in bold.

\begin{tabular}{lccccc}
\hline \hline $\begin{array}{l}\text { FePc@ } \\
\mathrm{Gr}\end{array}$ & $\begin{array}{c}\mathrm{E}_{a} \\
(\mathrm{eV})\end{array}$ & $\begin{array}{c}\mathrm{D}_{h} \\
(\AA)\end{array}$ & $\begin{array}{c}\text { CoPc@ } \\
\mathrm{Gr}\end{array}$ & $\begin{array}{c}\mathrm{E}_{a} \\
(\mathrm{eV})\end{array}$ & $\begin{array}{c}\mathrm{D}_{h} \\
(\AA)\end{array}$ \\
\hline Top & 3.38 & 3.37 & Top & $\mathbf{3 . 3 9}$ & $\mathbf{3 . 3 9}$ \\
Bridge & $\mathbf{3 . 4 0}$ & $\mathbf{3 . 3 4}$ & Bridge & 3.38 & 3.34 \\
Hex & 3.36 & 3.43 & Hex & 3.35 & 3.43 \\
\hline \hline
\end{tabular}


from the physisorption of iron porphyrin (FeP) molecule on graphene, where the energetically favorable physisorption position is on the top of $\mathrm{C}$ atom [8]. This can be attributed to the larger macrocycle in $\mathrm{FePc}$ as compared to that of in $\mathrm{FeP}$ and, hence, stronger vdW interaction. However, for CoPc, the most stable physisorption site on graphene is the top site, where the metal atom is on top of a $\mathrm{C}$ atom. This is similar to cobalt porphyrin $(\mathrm{CoP})$ physisorption on graphene/ $\mathrm{Ni}$ observed previously [35]. However, for both the physisorbed molecules, the difference in physisorption energies for different configurations is quite small (see Table II).

A comparison of results listed in Table I and Table II shows an interesting contrast between the two cases of physisorption of the $M \mathrm{Pc}$ molecules on the $\mathrm{MoS}_{2}$ and the graphene. It can be clearly observed that the energies of the MPc physisorption are quite high for the $\mathrm{MoS}_{2}$ as compared to that of the graphene. These are due to a strong interaction between the metal $d_{\pi}$ states with the $\mathrm{S} p_{z}$ states of $\mathrm{MoS}_{2}$ (see detailed discussions on density of states in Sec. III B). On graphene, the interaction between metal $d_{\pi}$ states and C $p_{z}$ states is relatively weak and hence the physisorption energies are much lower. The different strengths of interactions are also reflected on the vertical distances of $M \mathrm{Pc}$ molecules from the $\mathrm{MoS}_{2}$ and graphene surface. For energetically most favorable physisorption position, the vertical distances between the molecule metal centers and graphene are $\sim 0.20 \AA$ higher as compared to that of the vertical distances between the molecule metal center and the $\mathrm{MoS}_{2}$. The analyses of the structures also show that, unlike the $\mathrm{MoS}_{2}$, the physisorbed molecules on the graphene remain flat.

It is also interesting to note that the physisorption energy differences between different positions of the MPc molecules on the graphene are quite small $(\sim 0.02 \mathrm{eV})$. However, on the $\mathrm{MoS}_{2}$, the physisorption energy difference between the most stable physisorption site and the next probable site is substantially higher as compared to that of the graphene $(\sim 0.14 \mathrm{eV})$. These are due to the fact that the graphene is planar and hence the interaction of the graphene with the physisorbed molecules will not change drastically. However, in the $\mathrm{MoS}_{2}$ surface, $\mathrm{S}$ and Mo atoms are in different layers and, $\mathrm{S}$ being more electronegative, interacts strongly with the physisorbed molecules.

Our analyses of the structural properties and physisorption energies conclude that the MPc molecules have weaker interaction with graphene surface, but they will be strongly physisorbed on $\mathrm{MoS}_{2}$ surface.

\section{B. Electronic structure}

In this subsection, we will discuss the electronic properties of the $M \mathrm{Pc}$ molecules physisorbed on $\mathrm{MoS}_{2}$ and graphene. We have used total, site, and $m_{l}$ projected density of states and charge densities for our analysis.

In the two panels of Figs. 2(a) and 2(b), respectively, we have shown the $m_{l}$ decomposed $d$ states of the $\mathrm{Fe}$ atom of the FePc molecule physisorbed on $\mathrm{MoS}_{2}$ and graphene. For comparison, $m_{l}$ decomposed $d$ states of the $\mathrm{Fe}$ atom from the gas phase FePc molecule are plotted in Fig. 2(c). In Fig. 2(a), we have also plotted the projected density of states of $p_{z}$ orbitals of $\mathrm{S}$ atoms of $\mathrm{MoS}_{2}$ beneath the FePc molecule.

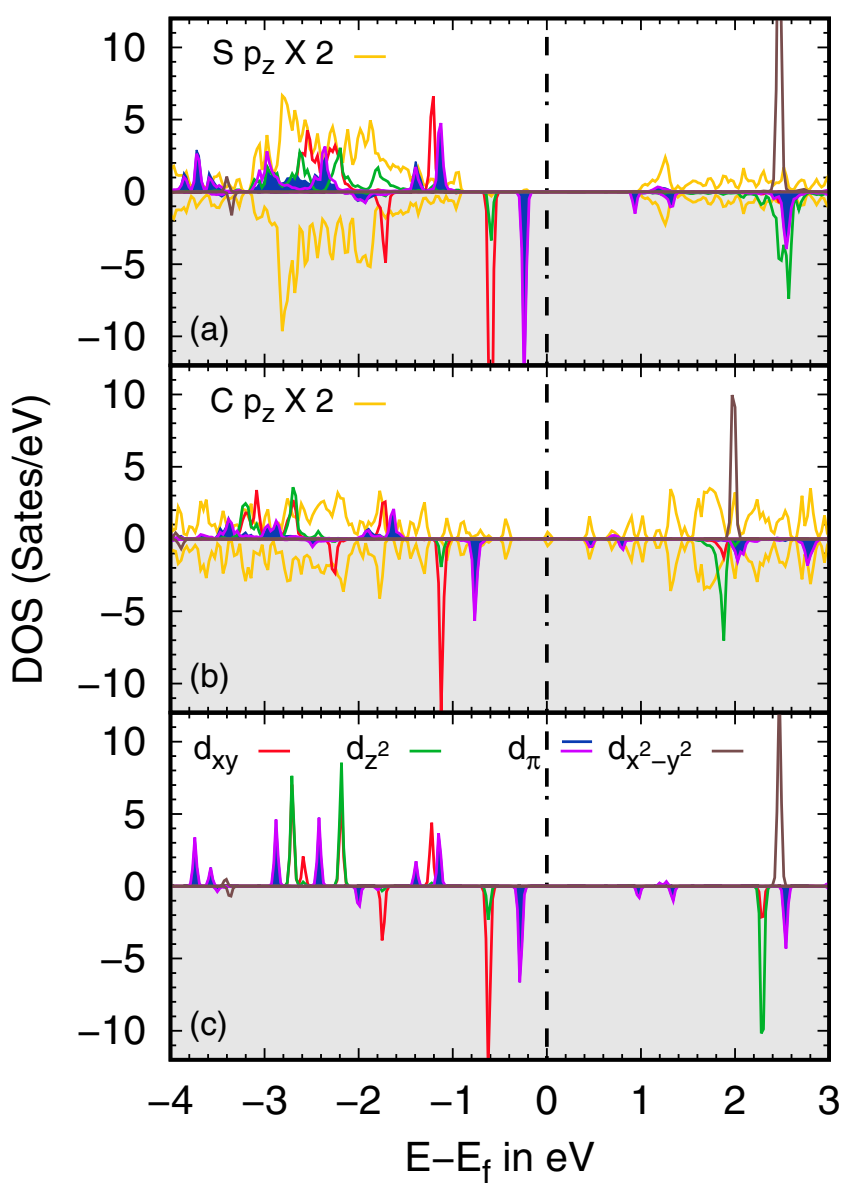

FIG. 2. Density of states plots for- (a) Fe $d$ orbitals of FePc physisorbed on $\mathrm{MoS}_{2}$ along with the $p_{z}$ orbitals of the $\mathrm{S}$ atoms beneath the molecule, (b) Fe $d$ orbitals of FePc physisorbed on graphene along with the $p_{z}$ orbitals of the $\mathrm{C}$ atoms beneath the molecule, and (c) Fe $d$ orbitals of gas phase FePc. The white and the grey areas represent the spin-up and spin-down channels of the DOS, respectively.

The $p_{z}$ orbitals of the $\mathrm{C}$ atoms of graphene below the $\mathrm{FePc}$ molecule are also plotted in Fig. 2(b). In Figs. 3(a)-3(c), we have plotted the similar quantities as described for Fig. 2, but for Co atom of the CoPc molecule.

From the analyses of projected density of states, it can be seen that the out-of-plane $d$ orbitals of the $\mathrm{Fe}$ atom become broadened after physisorption of the FePc molecule on $\mathrm{MoS}_{2}$ as compared to that of the gas phase [see Fig. 2(a)]. These are mainly seen on the occupied out-of-plane $d_{z^{2}}$ orbital as well as $d_{\pi}$ orbitals. These broadening of orbitals are mainly due to the strong interactions between out-of-plane $d$ orbitals of the physisorbed molecule and the $p_{z}$ orbitals of top layer $\mathrm{S}$ atoms of $\mathrm{MoS}_{2}$. In case of graphene, a similar broadening can also be seen [see Fig. 2(b)]. However, the interactions are relatively weak, which are evident from the smaller physisorption energies and larger vertical distances (cf. Tables I and II). Similar broadening of $d_{\pi}$ orbitals can also be observed for Co atom of $\mathrm{CoPc}$ molecule physisorbed on $\mathrm{MoS}_{2}$ and graphene [see Figs. 3(a)-3(b)]. However, for CoPc physisorption, the broadening of $d_{z^{2}}$ orbital of Co atom is significantly less as compared to that of the FePc physisorption. 


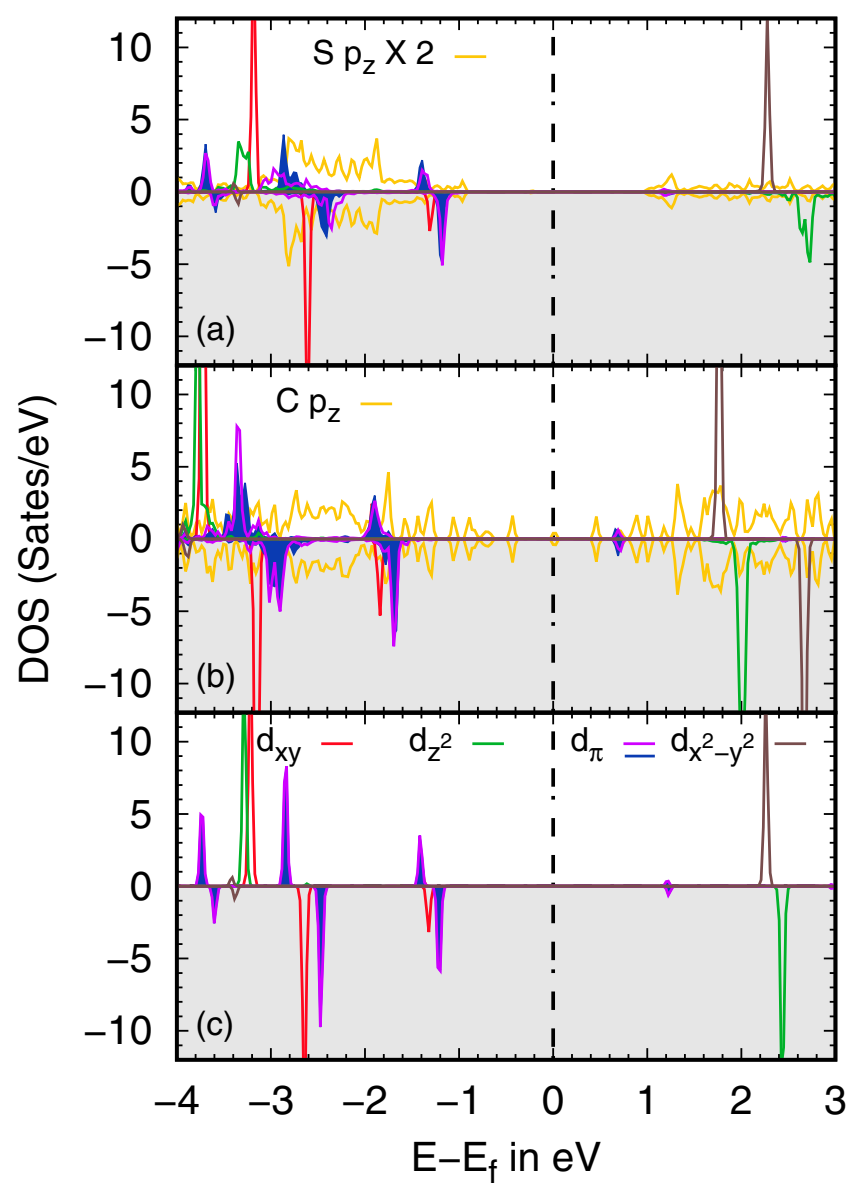

FIG. 3. Density of states plots for- (a) Co $d$ orbitals of CoPc physisorbed on $\mathrm{MoS}_{2}$ along with the $p_{z}$ orbitals of the $\mathrm{S}$ atoms beneath the molecule, (b) Co $d$ orbitals of CoPc physisorbed on graphene along with the $p_{z}$ orbitals of the $\mathrm{C}$ atoms beneath the molecule, and (c) Co $d$ orbitals of gas phase CoPc. The white and the grey areas represent the spin-up and spin-down channels of the DOS, respectively.

The total magnetic moments after physisorption on $\mathrm{MoS}_{2}$ and graphene are $2.0 \mu_{B}$ and $1.0 \mu_{B}$, respectively, for FePc and CoPc. For both molecules, the major part of the magnetic moments originate from the $d_{z^{2}}$ orbital of the metal atom. The physisorbed $\mathrm{FePc}$ has a spin configuration of $S=1$. This results from $b_{2 g}^{2}, e_{g}^{3}, a_{1 g}^{1}$ electronic configurations where $b_{2 g}\left(d_{x y}\right)$ orbital has an occupancy $2, e_{g}\left(d_{\pi}\right)$ orbital has an occupancy 3 and $a_{1 g}\left(d_{z^{2}}\right)$ orbital has an occupancy 1. However, for CoPc, the spin configuration is $S=1 / 2$, which results from $b_{2 g}^{2}, e_{g}^{4}, a_{1 g}^{1}$ electronic configuration where $b_{2 g}\left(d_{x y}\right)$ orbital has an occupancy $2, e_{g}\left(d_{\pi}\right)$ orbital has an occupancy 4 and $a_{1 g}\left(d_{z^{2}}\right)$ orbital has an occupancy 1 . The Bader charge analysis indicates that $\sim 0.09$ electrons are transferred per MPc molecule physisorbed on graphene. For physisorption on $\mathrm{MoS}_{2}$, the amounts of charge transfer are $\sim 0.004$ and $\sim 0.01$ electrons for FePc and CoPc molecule, respectively. These very small values indicate that there are hardly any charge transfers occur after the physisorption. Our calculations for the gas phase molecules show that the magnetic moments and the electronic configurations of $M \mathrm{Pc}$ molecules in the physisorbed situation do not change from the gas phase values. These are because the $d_{\pi}$ and $d_{z^{2}}$ orbitals are energetically positioned far below the Fermi energy. Thus, there is no significant hybridization with the surface, hence almost no transfer of electrons occurs. The $m_{l}-$ projected density of states corresponding to free molecule and molecule on surface differs slightly, but the orbital resolved occupations (and hence the magnetic moment) remain the same. The effect of the surface molecular interaction in molecular electronic structure is, hence, revealed in energy-resolved projected DOS but not in the quantities like orbital projected occupations or magnetic moment, which are energy integrated.

\section{Analysis of charge density difference}

To find out the charge accumulations due to the electronic interactions between the molecules and the surface, we have computed and analyzed the charge density differences for the composite $M \mathrm{Pc}$ molecules physisorbed on $\mathrm{MoS}_{2}$ /graphene systems with respect to the isolated $M P c$ molecules and pristine $\mathrm{MoS}_{2} /$ graphene surface. We have used the following formula for the calculation:

$$
\Delta \rho=\rho_{M \mathrm{Pc}} @ \mathrm{MoS}_{2} / \text { graphene }-\left(\rho_{\mathrm{MoS}_{2} / \text { graphene }}+\rho_{M \mathrm{Pc}}\right) .
$$

Here, $\rho_{M \mathrm{Pc}} @ \mathrm{MoS}_{2} /$ graphene, $\rho_{\mathrm{MoS}_{2} / \text { graphene }}$, and $\rho_{M \mathrm{Pc}}$ are the total charge densities of the composite physisorbed system, isolated $\mathrm{MoS}_{2} /$ graphene, and isolated $M \mathrm{Pc}$ molecules, respectively. For the isolated systems, we have fixed the geometries obtained from the optimized composite systems. In Figs. 4 and 5, we have shown these charge density difference plots for $\mathrm{FePc}$ and $\mathrm{CoPc}$ physisorbed systems, respectively (see Supplemental Material [36] for top and $360^{\circ}$ views of the charge density differences). From the analyses of the charge density difference plots, we see that a significant amount of charge accumulates on the whole $\mathrm{MoS}_{2}$ surface for the $M \mathrm{Pc}$ physisorption. These observations are consistent with charge density difference observed for physisorption of copper phthalocyanine molecule on mono layer $\mathrm{MoS}_{2}$ [25]. However, in the case of graphene, the accumulated charges are localized at the vicinity of the MPc molecules only. This large amount of charge accumulation on the $\mathrm{MoS}_{2}$ surface also indicates that there are strong vdW interactions $(\sim 0.13 \mathrm{eV} /$ atom higher vdW energy contribution) between the MPc molecules and the $\mathrm{MoS}_{2}$ surface as compared to that of the vdW interactions between the MPc molecules and the graphene.

\section{Spin dipole contribution}

For a low symmetry structure, deformations in the spin densities are expected to be large. These deformed spin densities lead to a large value of spin dipole moment $7\langle T\rangle$. The spin dipole moments are relevant for the XMCD measurements, where the measured effective moments contain both spin and spin-dipole moments. The spin dipole operator, $\mathrm{T}$, is defined as follows [37]:

$$
T=\sum_{i} Q^{(i)} s^{(i)}
$$

where, $Q^{(i)}$ is the quadrupolar tensor and can be described as

$$
Q_{\alpha \beta}^{(i)}=\delta_{\alpha \beta}-3 \hat{r}_{\alpha}^{(i)} \hat{r}_{\beta}^{(i)} .
$$




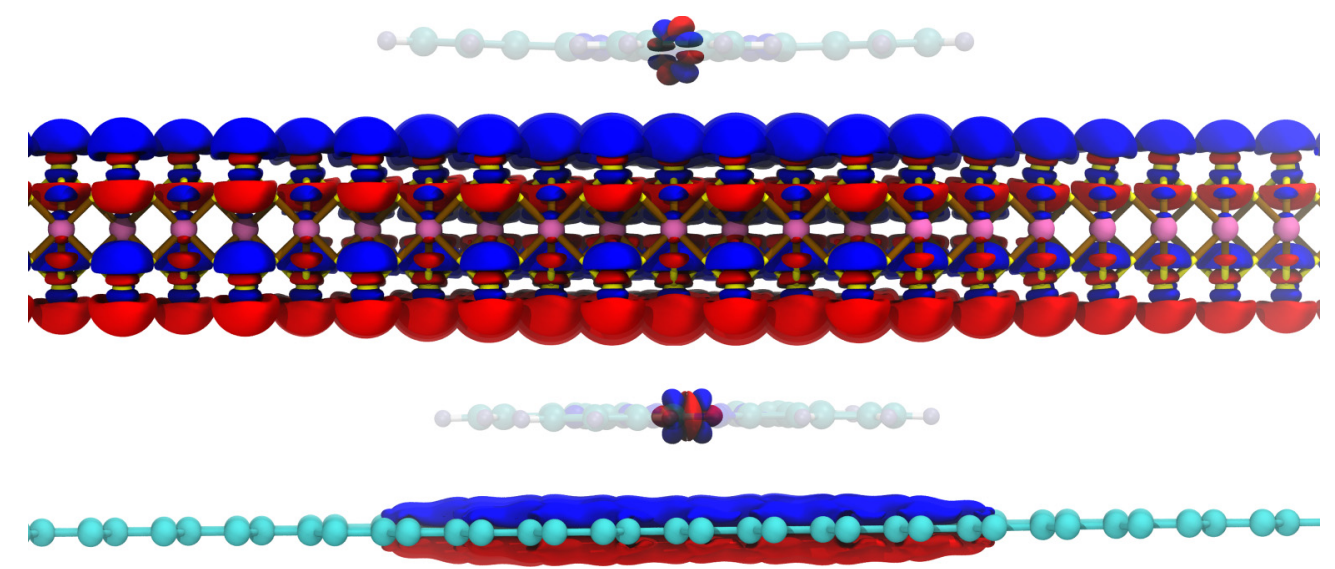

FIG. 4. Charge density difference plots between the $\mathrm{MoS}_{2} /$ graphene surface and FePc for the composite FePc on MoS $($ top panel) and $\mathrm{FePc}$ on graphene (bottom panel) system. The isosurfaces are plotted with one-tenth of the maximum isosurface values. Red (blue) colors denote positive (negative) charge density values. The FePc molecule is made transparent for easier viewing of the charge density difference in the molecule.

The components of $T$ can be written in the second quantized form as

$$
\begin{gathered}
T_{ \pm}=T_{x} \pm i T_{y}=\sum_{\gamma \gamma^{\prime}} T_{\gamma \gamma^{\prime}}^{ \pm} a_{\gamma}^{\dagger} a_{\gamma^{\prime}}, \\
T_{z}=\sum_{\gamma \gamma^{\prime}} T_{\gamma \gamma^{\prime}}^{z} a_{\gamma}^{\dagger} a_{\gamma^{\prime}},
\end{gathered}
$$

where $a_{\gamma}\left(a_{\gamma}^{\dagger}\right)$ are the annihilation (creation) operators. The matrix elements of $T_{ \pm}$and $T_{z}$ are as follows:

$$
\begin{gathered}
T_{\gamma \gamma^{\prime}}^{ \pm}=\left\langle\gamma\left|c_{0}^{2} s_{ \pm}-\sqrt{6} c_{ \pm 2}^{2} s_{\mp} \pm \sqrt{6} c_{ \pm 1}^{2} s_{z}\right| \gamma^{\prime}\right\rangle \\
T_{\gamma \gamma^{\prime}}^{z}=\left\langle\gamma\left|-\sqrt{\frac{3}{2}} c_{-1}^{2} s_{+}+\sqrt{\frac{3}{2}} c_{1}^{2} s_{-}-2 c_{0}^{2} s_{z}\right| \gamma^{\prime}\right\rangle
\end{gathered}
$$

where, $|\gamma\rangle=|\operatorname{lm}, \sigma\rangle$.

To calculate the spin dipole moments in $M P c$, we have followed the method prescribed by Freeman et al. [38] and performed density functional theory calculations including all the above-discussed effects of surface molecular interactions. The results of calculated spin dipole moments for the MPc gas phase molecules and the MPc molecules physisorbed on the $\mathrm{MoS}_{2}$ and the graphene are tabulated in Table III. For the $M \mathrm{Pc}$ molecules with transition metal atom center, the $z$ component of the spin dipole moment will be considered. As seen from the table, the calculated values of the $z$ component of the spin dipole moments $\left(7\left\langle T_{z}\right\rangle\right)$ are $-2.09 \mu_{B}$ and $-1.69 \mu_{B}$ for the FePc and the CoPc physisorbed on the monolayer $\mathrm{MoS}_{2}$, respectively. On the graphene, the values are $-2.05 \mu_{B}$ and $-1.70 \mu_{B}$ for the FePc and the CoPc, respectively. From our calculations, it is clear that the values of the spin dipole moments $\left(7\left\langle T_{z}\right\rangle\right)$ are very strong and they are opposite to the spin moment $\left(2\left\langle S_{z}\right\rangle\right)$. Hence, the effective spin moment defined as $S_{\text {eff }}=2\left\langle S_{z}\right\rangle+7\left\langle T_{z}\right\rangle$, will be reduced by a significant amount.

Our calculations also indicate that the comparative values of the spin dipole moments do not change significantly for both MPc molecules after physisorption on $\mathrm{MoS}_{2}$ or graphene as compared to that of the gas phase. The values of the spin

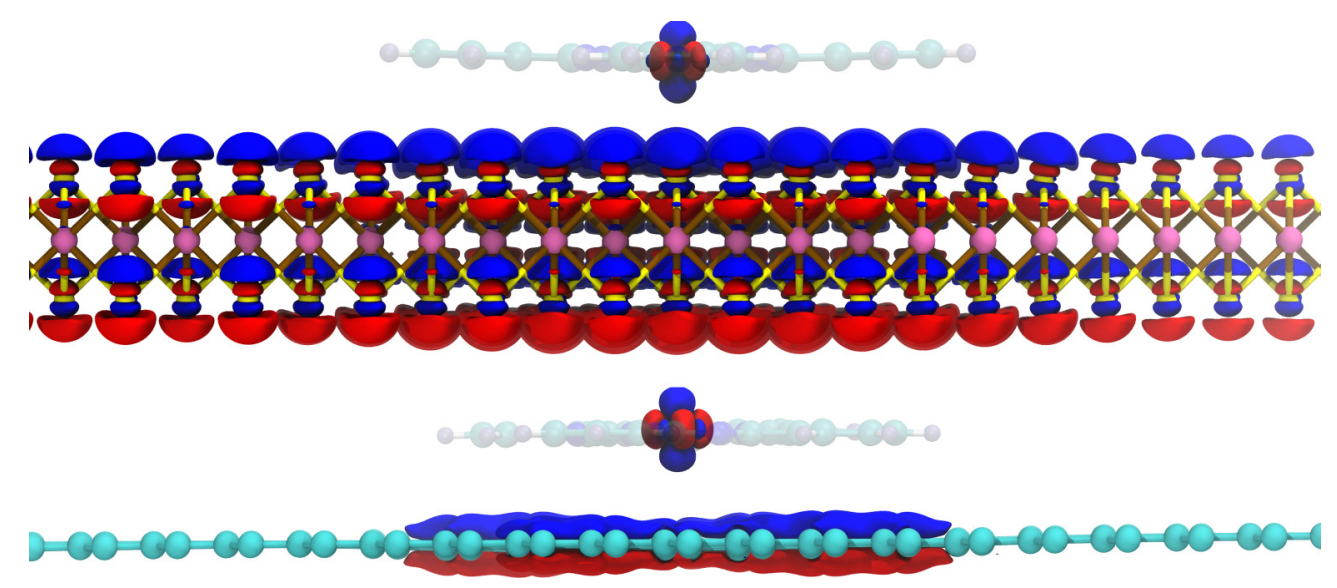

FIG. 5. Charge density difference plots between the $\mathrm{MoS}_{2} /$ graphene surface and CoPc for the composite CoPc on MoS $($ top panel) and $\mathrm{CoPc}$ on graphene (bottom panel) system. The isosurfaces are plotted with one-tenth of the maximum isosurface values. Red (blue) colors denote positive (negative) charge density values. The CoPc molecule is made transparent for easier viewing of the charge density difference in the molecule. 
TABLE III. Total spin magnetic moments $\left(2\left\langle S_{z}\right\rangle\right), z$ component of spin dipole moments $\left(7\left\langle\mathrm{~T}_{z}\right\rangle\right)$, and effective spin moments $\left(S_{\text {eff }}=\right.$ $\left.2\left\langle S_{z}\right\rangle+7\left\langle T_{z}\right\rangle\right)$ in the units of $\mu_{\mathrm{B}}$ for $M \mathrm{Pc}$ molecules physisorbed on monolayer $\mathrm{MoS}_{2}$ (top two rows), graphene (middle two rows), and gas phase $M P c$ (bottom two rows).

\begin{tabular}{lccc}
\hline \hline System & $2\left\langle S_{z}\right\rangle$ & $7\left\langle\mathrm{~T}_{z}\right\rangle$ & $S_{\text {eff }}$ \\
\hline FePc@ $\mathrm{MoS}_{2}$ & 2.0 & -2.09 & -0.09 \\
CoPc@ $\mathrm{MoS}_{2}$ & 1.0 & -1.69 & -0.69 \\
FePc@graphene & 2.0 & -2.05 & -0.05 \\
CoPc@graphene & 1.0 & -1.70 & -0.70 \\
FePc & 2.0 & -1.99 & 0.01 \\
CoPc & 1.0 & -1.70 & -0.70 \\
\hline \hline
\end{tabular}

dipole moments depend mainly on the electronic occupations of the $d$ orbitals of the metal atom center of MPc molecules. The analyses of density matrices show that the integrated quantities, such as the electronic occupations of the $d$ orbitals, do not change significantly due to the molecular physisorption situation as compared to that of the gas phase. Hence the changes in spin dipole moments from the gas phase are minimal.

\section{Orbital moments and magnetic anisotropy}

We have incorporated the spin-orbit coupling in the Hamiltonian of our DFT calculations to calculate orbital moments and magnetocrystalline anisotropy energy (MCA). We have used two different approaches to calculate the MCA (i) variational approach as implemented in VASP code and (ii) secondorder perturbation approach where the required energy eigenvalues and the corresponding orbital characters are obtained from scalar-relativistic Kohn-Sham DFT calculations. The MCA originates from the coupling between orbital and spin degrees of freedom. It can be expressed as follows:

$$
H_{S O}=\xi \mathbf{L} \cdot \mathbf{S}=\xi\left(L_{x} S_{x}+L_{y} S_{y}+L_{z} S_{z}\right),
$$

where $H_{S O}$ is the spin-orbit coupling Hamiltonian and $\xi$, the spin orbit coupling constant, is the radial average of $\xi(r)$ over the $3 d$ orbitals which can be defined as follows:

$$
\xi=\langle\xi(r)\rangle=\int R_{3 d}^{2}(r) \xi(r) r^{2} d r
$$

where $R_{3 d}(r)$ is the radial part of the $3 d$ wave function. Using the second order perturbation theory, one can write the spinorbit contribution to the energy as follows:

$$
E_{S O}=-\xi^{2} \sum_{u, o} \frac{[\langle u|\mathbf{L} \cdot \mathbf{S}| o\rangle\langle o|\mathbf{L} \cdot \mathbf{S}| u\rangle]}{E_{u}-E_{o}}
$$

In the above equation, $|o\rangle$ and $|u\rangle$ represent the weighted occupied and unoccupied states of Fe/Co $3 d$ orbitals. These occupied and unoccupied states are weighted by the occupations of respective $3 d$ orbitals, $|o / u\rangle=|l m, \sigma\rangle$. The orbital and spin operators are denoted by $\mathbf{L}$ and $\mathbf{S}$. $E_{o}$ and $E_{u}$ represent, respectively, the eigenvalues of occupied and unoccupied states which have been taken from the $a b$ initio calculations. The values of $\xi$ have been taken as $49.6 \mathrm{meV}$ and $63.8 \mathrm{meV}$ for $\mathrm{Fe}^{2+}$ and $\mathrm{Co}^{2+}$, respectively [39].
TABLE IV. MCA data within the second variation approach as implemented in VASP code, MCA $\left(\Delta E=E^{\text {hard }}-E^{\text {easy }}\right)$, and orbital moment $\left(\mu_{\text {orb }}\right)$ for $M$ Pc molecules physisorbed on monolayer $\mathrm{MoS}_{2}$ (top table), on graphene (middle table), and gas phase MPc (bottom table).

\begin{tabular}{lccc}
\hline \hline System & $\mu_{\text {orb }}$ & $\Delta E(\mu \mathrm{eV})$ & Easy axis \\
\hline FePc@ $\mathrm{MoS}_{2}$ & 0.012 & 52 & Out-of-plane \\
CoPc@ $\mathrm{MoS}_{2}$ & 0.036 & 75 & In-plane \\
System & $\mu_{\text {orb }}$ & $\Delta E(\mu \mathrm{eV})$ & Easy axis \\
\hline FePc@graphene & 0.012 & 48.5 & Out-of-plane \\
CoPc@ graphene & 0.037 & 76 & In-plane \\
System & $\mu_{\text {orb }}$ & $\Delta E(\mu \mathrm{eV})$ & Easy axis \\
\hline FePc & 0.012 & 30 & Out-of-plane \\
CoPc & 0.037 & 78 & In-plane \\
\hline \hline
\end{tabular}

From Eq. (11), it is clear that the spin-orbit coupling energy contribution increases when $E_{u}-E_{o}$ decreases. Therefore, the relative placement of the $3 d$ orbitals of the $\mathrm{Fe} / \mathrm{Co}$ will dictate the energy contribution to the spin-orbit coupling. The relative arrangement of the $3 d$ orbitals can be affected by the physisorption site of the $M \mathrm{Pc}$ on $\mathrm{MoS}_{2}$ or graphene. It is worth pointing out that the orbital angular momentum, via the term $\mathbf{L} \cdot \mathbf{S}$, lowers the spherical symmetry of the spin, and hence the geometric anisotropy also affects the magnetic anisotropy. However, the effects of geometrical symmetry lowering (owing to the surface molecular interaction) will be reflected in the nature and energy position of the molecular levels. The relative contributions of different transition metal $d$ orbitals and ligand $p$ orbitals in a molecular level will change if the symmetry is changed. These effects are already incorporated in our calculations by considering the projected occupations, which appear in the numerator along with the change in energy positions in the denominator of Eq. (11) for calculating energy using second-order perturbation theory. A chemisorbed situation can induce such significant changes, e. g., enhancement of metal-N bond length, spin-state change, etc. $[7,8]$. However, due to weak interactions in the case of physisorption, these effects are quite small.

We have tabulated the results obtained using the variational approach and the second-order perturbation approach in Tables IV and V, respectively. We have reported the orbital

TABLE V. MCA data within the second order perturbation approach, magnetic anisotropy energy $\left(\Delta E=E^{\text {hard }}-E^{\text {easy }}\right)$, and orbital moment $\left(\mu_{\mathrm{orb}}\right)$ for $M \mathrm{Pc}$ molecules physisorbed on monolayer $\mathrm{MoS}_{2}$ (top table), on graphene (middle table), and gas phase $M \mathrm{Pc}$ (bottom table).

\begin{tabular}{lccc}
\hline \hline System & $\mu_{\text {orb }}$ & $\Delta E(\mu \mathrm{eV})$ & Easy axis \\
\hline FePc@ $\mathrm{MoS}_{2}$ & 0.019 & 103 & Out-of-plane \\
CoPc@ $\mathrm{MoS}_{2}$ & 0.028 & 58 & In-plane \\
System & $\mu_{\text {orb }}$ & $\Delta E(\mu \mathrm{eV})$ & Easy axis \\
\hline FePc@graphene & 0.024 & 107.8 & Out-of-plane \\
CoPc@graphene & 0.030 & 60.2 & In-plane \\
System & $\mu_{\text {orb }}$ & $\Delta E(\mu \mathrm{eV})$ & Easy axis \\
\hline FePc & 0.026 & 98 & Out-of-plane \\
CoPc & 0.028 & 60 & In-plane \\
\hline \hline
\end{tabular}


magnetic moments, magnetic anisotropy energies along with the information about the easy axis in Tables IV and V. Both approaches mentioned before give similar qualitative results. As seen from Table IV, the orbital moments do not change significantly in the physisorbed systems. The analyses of $m_{l}$ projected density of states (see Figs. 2-3) indicate that, although the $d$ orbitals below the Fermi levels are broadened in the physisorbed system compared to the gas phase, the band centers remain the same. Hence the orbital moments remain almost similar. The relative positions of $d$ orbitals of the metal center in the $M P c$ molecules do not change significantly from gas phase into the physisorbed phase. Therefore, the transition matrices are similar and hence the MCAs are similar in values. Our results indicate that while for $\mathrm{CoPc}$, the easy axis of magnetization is in-plane, the easy axis is out-of-plane for FePc. However, it is important to note that the DFT+U calculations with static correlations lack correct estimation of MCA and one would require further advanced approaches beyond DFT to incorporate many body effects with dynamical correlations for correct estimations of MCA [40].

\section{Work function}

One of the important features of adsorption is the change of work function of the substrates. In the present context, this can be achieved by the decoration of the 2D substrates by the molecules, which may be useful for designing nano devices. To investigate possible changes in work functions $(\Phi)$ of the $\mathrm{MoS}_{2}$ and graphene due to the $M \mathrm{Pc}$ physisorption, we have calculated the work functions of the pristine monolayer $\mathrm{MoS}_{2}$, graphene, and $M \mathrm{Pc}$ physisorbed on $\mathrm{MoS}_{2}$ and graphene. The work function $\Phi$ is defined as the minimum energy required to remove an electron from a material to the vacuum. Hence, $\Phi$ can be defined as follows:

$$
\Phi=V(\infty)-E_{f},
$$

where, $V(\infty)$ is the self-consistent electrostatic potential in the vacuum far from the surface and $E_{f}$ is the Fermi energy, which is calculated from a ground-state self-consistent calculation. Here, the top of the valence band has been taken as the Fermi energy. The surface work functions were calculated by applying the Neugebauer-Scheffler dipole correction [41] for the direction perpendicular to the surface. The electrostatic potential is obtained as a function of $z(V(z))$ by averaging the self-consistent potential parallel to the surface, i.e., in the $x y$ plane. Then the potential $V(\infty)$ is approximated as the value of potential at the vacuum layer, where $V(z)$ is reaching its asymptotic limit.

In Table VI, we have tabulated the calculated values of $\Phi$ for the above-mentioned cases. The value of $\Phi$ for monolayer

TABLE VI. Comparison of work function $(\Phi)$ values for monolayer $\mathrm{MoS}_{2}$, graphene and $M \mathrm{Pc}$ molecules physisorbed on $\mathrm{MoS}_{2}$ and graphene.

\begin{tabular}{lccc}
\hline \hline System & $\Phi(\mathrm{eV})$ & System & $\Phi(\mathrm{eV})$ \\
$\mathrm{MoS}_{2}(1 \mathrm{~L})$ & 5.94 & Graphene $(1 \mathrm{~L})$ & 4.23 \\
\hline FePc@MoS & 4.94 & FePc@Graphene & 4.25 \\
CoPc@MoS & 5.17 & CoPc@Graphene & 4.25 \\
\hline \hline
\end{tabular}

graphene is $4.23 \mathrm{eV}$. This value is quite consistent with previous published result [42]. For $\mathrm{MoS}_{2}$, the computed value of $\Phi$ is $5.94 \mathrm{eV}$, which is also quite similar to earlier published result [43]. It is evident from our calculations that the values of $\Phi$ do not change significantly after MPc physisorptions on graphene. However, the work function values decrease substantially $(\sim 1 \mathrm{eV}$ for FePc and $\sim 0.8 \mathrm{eV}$ for CoPc) due to $M \mathrm{Pc}$ physisorptions on $\mathrm{MoS}_{2}$. Therefore, we conclude that it may be possible to tune the $\Phi$ of $\mathrm{MoS}_{2}$ by $M \mathrm{Pc}$ physisorption, which opens up the possibility of using these materials in electronic and optoelectronic devices for applications in fluorescence imaging, solar cells, and photocatalysis.

\section{CONCLUSIONS}

In conclusion, we have performed detailed investigations of electronic and magnetic properties of $M \mathrm{Pc}(M=\mathrm{Fe}, \mathrm{Co})$ physisorption on $\mathrm{MoS}_{2}$ and graphene using density functional theory along with Coulomb correlation for the metal $d$ electrons. We have considered various possible physisorption sites both on $\mathrm{MoS}_{2}$ and on graphene to find out energetically most favorable configurations. We have found that MPc molecules are physisorbed strongly on the $\mathrm{MoS}_{2}$ as compared to that of the graphene. The most stable physisorption site on $\mathrm{MoS}_{2}$ is when the metal atom center of a MPc molecule resides on top of a $\mathrm{S}$ atom. The other physisorption sites have much higher energy $(\sim 0.14 \mathrm{eV})$. However, on graphene, the physisorption energies are very similar for different physisorption sites. The out-of-plane $d$ orbitals of the metal centers interacts more strongly with the out-of-plane orbitals from the $\mathrm{MoS}_{2}$ as compared to that of the graphene. The magnetic moment arises from $d_{z^{2}}$ orbital of the metal atom center of the molecules. The $M \mathrm{Pc}$ physisorptions on the $\mathrm{MoS}_{2}$ reduce the work function by $\sim 1 \mathrm{eV}$ whereas it does not change much in the case of physisorption on the graphene. The MPc physisorptions cause the large spin dipole moments opposite to the spin moments causing huge reduction of the effective moments, which can be measured by XMCD experiments. Our calculations of magnetic anisotropy energies using both variational and second-order perturbation approaches indicate no significant changes in the magnetic anisotropy energy values after physisorption of the MPc molecules. An out-of-plane magnetic anisotropy can be observed in the case of FePc whereas for $\mathrm{CoPc}$, it is in-plane. In summary, our theoretical predictions indicate that the hybrid organic-inorganic interfaces consisting of metal centered phthalocyanine molecules and 2D $\mathrm{MoS}_{2}$ are very interesting choices of system for useful applications.

\section{ACKNOWLEDGMENTS}

This work is supported by the project grant (2016-05366) and Swedish Research Links programme grant (2017-05447) from the Swedish Research Council awarded to B.S. We gratefully acknowledge supercomputing time allocation by the Swedish National Infrastructure for Computing (SNIC) and PRACE-2IP project 'CHARTERED2' resource Salomon cluster based in Czech Republic at the IT4Innovations for performing the computations. S.H. acknowledges fruitful discussions with Souvik Paul and Vivekanand Shukla. Structural figures are generated using VMD [44]. 
[1] Y. Wang, J. Kröger, R. Berndt, and W. A. Hofer, J. Am. Chem. Soc. 131, 3639 (2009).

[2] J. Repp, G. Meyer, F. E. Olsson, and M. Persson, Science 305, 493 (2004).

[3] T. Komeda, H. Isshiki, J. Liu, Y.-F. Zhang, N. Lorente, K. Katoh, B. K. Breedlove, and M. Yamashita, Nat. Comm. 2, 217 (2011).

[4] A. Stróżecka, M. Soriano, J. I. Pascual, and J. J. Palacios, Phys. Rev. Lett. 109, 147202 (2012).

[5] M. G. Betti, P. Gargiani, R. Frisenda, R. Biagi, A. Cossaro, A. Verdini, L. Floreano, and C. Mariani, J. Phys. Chem. C 114, 21638 (2010).

[6] S. Bhandary, S. Ghosh, H. Herper, H. Wende, O. Eriksson, and B. Sanyal, Phys. Rev. Lett. 107, 257202 (2011).

[7] S. Bhandary, B. Brena, P. M. Panchmatia, I. Brumboiu, M. Bernien, C. Weis, B. Krumme, C. Etz, W. Kuch, H. Wende, O. Eriksson, and B. Sanyal, Phys. Rev. B 88, 024401 (2013).

[8] S. Bhandary, O. Eriksson, and B. Sanyal, Sci. Rep. 3, 3405 (2013).

[9] H. C. Herper, M. Bernien, S. Bhandary, C. F. Hermanns, A. Krüger, J. Miguel, C. Weis, C. Schmitz-Antoniak, B. Krumme, D. Bovenschen, C. Tieg, B. Sanyal, E. Weschke, C. Czekelius, W. Kuch, H. Wende, and O. Eriksson, Phys. Rev. B 87, 174425 (2013).

[10] C. Wäckerlin, D. Chylarecka, A. Kleibert, K. Müller, C. Iacovita, F. Nolting, T. Jung, and N. Ballav, Nat. Commun. 1, 61 (2010).

[11] L. Liu, K. Yang, Y. Jiang, B. Song, W. Xiao, L. Li, H. Zhou, Y. Wang, S. Du, M. Ouyang, W. Hofer, A. Castro Neto, and H.-J. Gao, Sci. Rep. 3, 1210 (2013).

[12] A. H. C. Neto, F. Guinea, N. M. R. Peres, K. S. Novoselov, and A. K. Geim, Rev. Mod. Phys 81, 109 (2009).

[13] A. K. Geim and K. Novoselov, Nat. Mater. 6, 183 (2007).

[14] A. K. Geim, Science 324, 1530 (2009).

[15] D. Xie, Chem. Commun. 52, 9418 (2016).

[16] A. Kudo and Y. Miseki, Chem. Soc. Rev. 38, 253 (2009).

[17] Y. Li, Y.-L. Li, C. M. Araujo, W. Luo, and R. Ahuja, Catal. Sci. Technol. 3, 2214 (2013).

[18] Q. H. Wang, K. Kalantar-Zadeh, A. Kis, J. N. Coleman, and M. S. Strano, Nature Nanotech. 7, 699 (2012).

[19] S. S. Chou, B. Kaehr, J. Kim, B. M. Foley, M. De, P. E. Hopkins, J. Huang, C. J. Brinker, and V. P. Dravid, Angew. Chem., Int. Ed. 52, 4160 (2013).

[20] T. Liu, C. Wang, W. Cui, H. Gong, C. Liang, X. Shi, Z. Li, B. Sun, and Z. Liu, Nanoscale 6, 11219 (2014).

[21] X. Yang, J. Li, T. Liang, C. Ma, Y. Zhang, H. Chen, N. Hanagata, H. Su, and M. Xu, Nanoscale 6, 10126 (2014).
[22] S. Haldar, H. Vovusha, M. K. Yadav, O. Eriksson, and B. Sanyal, Phys. Rev. B 92, 235408 (2015).

[23] J.-T. Liu, T.-B. Wang, X.-J. Li, and N.-H. Liu, J. Appl. Phys. 115, 193511 (2014).

[24] Y. Jing, X. Tan, Z. Zhou, and P. Shen, J. Mater. Chem. A 2, 16892 (2014).

[25] P. Choudhury, L. Ravavarapu, R. Dekle, and S. Chowdhury, J. Phys. Chem. C 121, 2959 (2017).

[26] G. Kresse and J. Furthmüller, Phys. Rev. B 54, 11169 (1996).

[27] J. P. Perdew, K. Burke, and M. Ernzerhof, Phys. Rev. Lett. 77, 3865 (1996).

[28] J. P. Perdew, K. Burke, and M. Ernzerhof, Phys. Rev. Lett. 78, 1396 (1997).

[29] S. L. Dudarev, G. A. Botton, S. Y. Savrasov, C. J. Humphreys, and A. P. Sutton, Phys. Rev. B 57, 1505 (1998).

[30] I. V. Solovyev, P. H. Dederichs, and V. I. Anisimov, Phys. Rev. B 50, 16861 (1994).

[31] V. I. Anisimov, F. Aryasetiawan, and A. I. Lichtenstein, J. Phys. Condens. Matter 9, 767 (1997).

[32] T. O. Wehling, A. I. Lichtenstein, and M. I. Katsnelson, Phys. Rev. B 84, 235110 (2011).

[33] T. Eelbo, M. Waśniowska, P. Thakur, M. Gyamfi, B. Sachs, T. O. Wehling, S. Forti, U. Starke, C. Tieg, A. I. Lichtenstein, and R. Wiesendanger, Phys. Rev. Lett. 110, 136804 (2013).

[34] A. Tkatchenko and M. Scheffler, Phys. Rev. Lett. 102, 073005 (2009).

[35] D. Klar, S. Bhandary, A. Candini, L. Joly, P. Ohresser, S. Klyatskaya, M. Schleberger, M. Ruben, M. Affronte, O. Eriksson, B. Sanyal, and H. Wende, Phys. Rev. B 89, 144411 (2014).

[36] See Supplemental Material at http://link.aps.org/supplemental/ 10.1103/PhysRevB.98.085440 for top and $360^{\circ}$ views of the charge density differences.

[37] T. Oguchi and T. Shishidou, Phys. Rev. B 70, 024412 (2004).

[38] R. Wu and A. J. Freeman, Phys. Rev. Lett. 73, 1994 (1994).

[39] G. M. Cole Jr. and B. B. Garrett, Inorg. Chem. 9, 1898 (1970).

[40] S. Bhandary, M. Schüler, P. Thunström, I. di Marco, B. Brena, O. Eriksson, T. Wehling, and B. Sanyal, Phys. Rev. B 93, 155158 (2016).

[41] J. Neugebauer and M. Scheffler, Phys. Rev. B 46, 16067 (1992).

[42] K. C. Kwon, K. S. Choi, and S. Y. Kim, Adv. Funct. Mater. 22, 4724 (2012).

[43] N. A. Lanzillo, A. J. Simbeck, and S. K. Nayak, J. Phys. Condens. Matter 27, 175501 (2015).

[44] W. Humphrey, A. Dalke, and K. Schulten, J. Mol. Graph. 14, 33 (1996). 